\title{
Patients with advanced cancer: when, why, and how to refer to palliative care services
}

C. Courteau MD, ${ }^{*}$ G. Chaput BA MD MA CAC(Pall Med) ${ }_{1}^{+\neq}$L. Musgrave MD, ${ }^{\ddagger}$ and A. Khadoury MD CAC(Pall Med $)^{\ddagger}$

\section{ABSTRACT}

Palliative care (PC) is a fundamental component of the cancer care trajectory. Its primary focus is on "the quality of life of people who have a life-threatening illness, and includes pain and symptom management, skilled psychosocial, emotional and spiritual support" to patients and loved ones. Palliative care includes, but is not limited to, end-of-life care. The benefits of early introduction of PC services in the care trajectory of patients with advanced cancer are well known, as indicated by improved quality of life, satisfaction with care, and a potential for increased survival. In turn, early referral of patients with advanced cancer to PC services is strongly recommended.

So when, how, and why should patients with advanced cancer be referred to PC services? In this article, we summarize evidence to address these questions about early PC referral:

What are the known benefits?

What is the "ideal" PC referral timing?

What are the barriers?

Which strategies can optimize integration of PC into oncology care?

Which communication tools can facilitate skillful introduction of PC to patients?

Key Words Palliative care referral, benefits, advanced cancer, communication skills

Curr Oncol. 2018 Dec;25(6):403-408

www.current-oncology.com

\section{INTRODUCTION}

Palliative care (PC) focuses on "the quality of life of people who have a life-threatening illness, and includes symptom management, skilled psychosocial and spiritual support" to patients and loved ones ${ }^{1}$. Palliative care has been found to enhance quality of life and satisfaction with care for patients with advanced cancer, to reduce chemotherapy use near the end of life, and potentially to increase survival ${ }^{2-5}$. Nonetheless, suboptimal PC referral remains a barrier to high-quality care in advanced cancer ${ }^{6}$. An authoritative report stated that two thirds of patients requiring PC services accessed them only during their final hospitalization ${ }^{6}$. Given its associated benefits ${ }^{2,3}$, early PC referral for patients with advanced cancer is strongly recommended?

In this article, we address these questions about PC referral:

What are the known benefits?

What is the "ideal" PC referral timing?

What are the barriers?
Which strategies can optimize integration of PC into oncology care?

Which communication tools can facilitate skillful introduction of PC to patients?

\section{QUESTIONS ABOUT EARLY PC REFERRAL}

\section{Benefits}

The exponential advances made in cancer treatment do not appear to have been matched by equivalent advances in supportive interventions, leaving patients with greater psychological and physical symptom burdens from their cancer therapies ${ }^{8}$.

This series is brought to you in partnership with the Canadian Association of General Practitioners in Oncology.

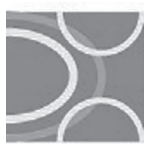

THE CANADIAN ASSOCIATION OF GENERAL PRACTITIONERS IN ONCOLOGY 
Oncologists likely feel genuine concern for the psychological well-being of their patients, but findings suggest that they lack the time to comprehensively screen for distress ${ }^{9}$. Early integration of PC might not only improve physical symptom control, but also enhance psychological health ${ }^{5,10}$. Early referral to PC for patients with advanced cancer has also been associated with enhanced quality of life by increasing the patient's understanding of their disease and anticipated prognosis, and facilitating coping and advance care planning ${ }^{11-13}$. Moreover, a recent report from the Canadian Partnership Against Cancer indicated that nearly 1 in 4 cancer patients experience 2 or more hospital admissions in the last 4 weeks of life, suggesting a need for an improved process of referral to PC services ${ }^{6}$. Evidence has shown that early Pc referral is associated with decreased use of chemotherapy and fewer hospitalizations near the end of life ${ }^{4,12}$. Worthy of mention is that fact that no harmful outcomes have been identified from early involvement of the PC team ${ }^{7}$. Integration of interdisciplinary PC services into the routine oncologic care of patients with advanced cancer is therefore recommended ${ }^{7}$.

\section{"Ideal" PC Referral Timing}

Several variables have been used to determine the most opportune time to refer patients to PC services, including cancer trajectory, disease extent, response to treatment or lack thereof, and anticipated prognosis.

With respect to cancer trajectory, the World Health Organization describes PC as "applicable early in the course of illness, in conjunction with other therapies that are intended to prolong life" 14 . Being poorly defined, the term "early" can lead to differences in interpretation about when PC referral is warranted. Similarly, considerable variation in the definition of the term "early" has been described, ranging from time of diagnosis of advanced disease, to shortly after or upon failure or discontinuation of curative treatments, to the period just before expected death (in months) ${ }^{15-18}$. Those variations could contribute to delays in the more prompt integration of PC services into patient care.

Another described marker for PC referral is disease extent. The U.S. National Cancer Institute defines advanced cancer as "cancer that has spread to other places in the body and usually cannot be cured or controlled with treatment" 19 . That definition has its limitations, in that certain malignancies (such as brain cancers) are associated with poor prognosis even in the absence of distant metastases. Likewise, given that decreased rates of aggressive treatment have been reported to be a benefit of early pc referral, the use of treatment failure or discontinuation as a referral criterion appears to be unsuitable.

Lastly, estimating the prognosis of cancer patients can be an imprecise and ambiguous process, with clinicians tending to overestimate survival ${ }^{20}$. Making use ofanticipated prognosis such as "less than 3 months" as an indicator for PC referral is therefore unreliable. The American Society of Clinical Oncology's recently published guideline about the integration of palliative care into standard oncology care recommends that "inpatients and outpatients with advanced cancer should receive dedicated palliative care services, early in the disease course, concurrent with active treatment" ${ }^{\text {" }}$. Referral to Pc for patients with advanced cancer should therefore occur as early as possible in the cancer trajectory, rather than be reserved until the last days of life.

\section{Barriers}

In addition to heterogeneity in PC delivery models $s^{3,21,22}$, specific patient-flow obstacles have been described as barriers to PC referral. Those obstacles include lack of referral criteria and clearly defined timing for PC referrals ${ }^{23,24}$.

Palliative care is a unique field, in that referrals are seldom based on objective criteria-contrary to, for example, an identified gastrointestinal bleed as a clear criterion for gastroenterology consultation. Rather, PC referrals are heavily influenced by patient need ${ }^{24}$. Although anticipated short prognosis has been reported to be an objective PC referral criterion, it has evident flaws, given that patients are often referred too late in the course of their illness ${ }^{25}$. Health provider-related obstacles have also been identified as barriers to PC referral. Those obstacles include the perception that PC is a service that exclusively provides end-of-life care ${ }^{26}$ and a disinclination on the part of oncologists and other professionals to refer patients to $\mathrm{PC}^{27}$. Moreover, physician reluctance to engage in discussions about expected prognosis with patients also contributes to delayed PC referral ${ }^{26}$.

In addition to end-of-life care, PC provides a wide array of services, including cancer treatment-related symptom management and psychosocial interventions, which have been shown to benefit patients and family members alike ${ }^{2,28,29}$. Further educational initiatives targeting oncologists and cancer care professionals are therefore urgently needed and show promise in optimizing access to PC services for patients ${ }^{27,30}$.

\section{Strategies}

Creating standardized referral pathways, classifying oncology patients into "low versus high" categories with respect to symptom burden, and adopting an integrated cancer care delivery model have been proposed as strategies to improve referral to PC services for patients. The establishment of standardized PC referral pathways to optimize patient flow has been widely recommended ${ }^{23,24,31}$. Hui et $a l .{ }^{24}$ identified the following 6 recurrent $P C$ referral criteria as benchmarks toward the development of standardized referral pathways: physical symptoms, disease trajectory, prognosis, performance status, psychosocial distress, and end-of-life care planning. Advocacy for clearly defined PC referral criteria as a means to facilitate objective assessment of programs and to guide policymaking has been described ${ }^{24}$. Referral guidelines should take into consideration specific characteristics, given that recent studies have highlighted benefits of PC referral based on the patient's cancer type, sex, and age ${ }^{9,32}$. Lastly, for the referral pathways to be effective, routine and seriated evaluations of the patient's symptoms and consideration for their disease course appear to be of paramount importance ${ }^{23,31}$.

Moreover, categorizing individuals as either low- or high-burden patients could be another strategy for referring patients to PC services when the existing infrastructure might not be capable of receiving universal early PC referrals ${ }^{33}$. "High-burden patients" [those experiencing treatment failure, physical or emotional distress (or both), 
and interpersonal conflicts] might benefit most from early PC involvement and could therefore be prioritized for referral $^{33,34}$. However, caution should be exercised when attempting to categorize patients in such a manner, given that "high-burden, low-burden" criteria would fail to identify patients with earlier-stage cancer requiring a referral to PC services ${ }^{22}$.

Lastly, integrated cancer care models appear most promising in introducing PC services early in the disease trajectory. Bruera and $\mathrm{Hui}^{34}$ described three conceptual models to optimize integration of PC services in oncology care: the oncologist solo-practice model, in which the oncologist attempts to deliver the entirety of cancer management, including supportive and palliative interventions; the congress practice model, in which several consultants are independently involved in a patient's care and which is associated with risks of costly and fragmented care; and the integrated care model, in which oncologists involve PC teams early on to collaborate closely in patient care. The integrated care model allows for the simultaneous delivery of cancer treatments and optimal management of cancer-related symptoms and problems, which are both essential and complementary to comprehensive cancer care. However, implementation of the integrated care model into the clinical setting can be limited should its adoption by the referring physicians be suboptimal ${ }^{34}$. Given that PC is a critical component of care that focuses on quality-of-life concerns and caregoal preferences, all efforts should therefore be made to integrate outpatient and inpatient PC services into traditional oncology care ${ }^{7}$.

\section{Communication Tools}

Given the nature of their profession, physicians are routinely tasked with disclosing unfavourable health information to patients ${ }^{35}$. Disclosing bad news can be a demanding responsibility, particularly in cases in which treatment options are limited or no longer available ${ }^{36}$. Oncology providers often have apprehensions about discussing PC referral with their patients, because they fear that such referral might be perceived as a sign of hopelessness or might cause depression $^{37}$. Nonetheless, professional ethics and legal obligations oblige physicians to inform patients about any information they request about their disease, treatment plan, and prognosis. Moreover, providing accurate information about prognosis and care plans to patients, whether positive or negative, does not influence hope in patients with advanced cancer ${ }^{37}$. On the contrary, evidence has shown a patient preference to be informed about their anticipated prognosis ${ }^{37}$, and that awareness of prognosis is associated with improved coping and future planning, and increased trust and satisfaction with care ${ }^{18-20}$. That said, the manner and ways in which difficult news is related to patients are of critical significance. To skillfully introduce PC to patients, the application of communication tools such as the use of metaphors and communication models can be helpful in guiding difficult conversations associated with PC referrals.

Core communication tactics such as empathic words and active listening can be supplemented with metaphors to facilitate end-of-life conversations ${ }^{38}$. When initiating a conversation about PC service referral with a patient, the "taking a road trip" metaphor can be helpful (Figure 1). In the metaphor, the cancer trajectory is compared to taking a road trip, and the goal is to arrive at destination-that is, to treat the cancer. Some drivers focus solely on arriving at their destination and do not preoccupy themselves with comfort and safety features such as the seatbelt, spare tire, or seat cushion. Other drivers benefit from those features along the ride, such that they can get to their destination in a safer, more comfortable manner. Drivers who opt for the comfort and safety features are better equipped to pursue their goals. Similarly, Pc teams can provide physical and psychosocial symptom control by acting as a cushion to help patients throughout their cancer journey. Palliative care services can be compared to robust roadside assistance program that provides security during a road trip ${ }^{38}$. When skillfully used, the metaphor can enhance a patient's understanding and guide future care planning ${ }^{38}$.

Another communication tool useful for facilitating the introduction of PC to patients is the brief and practical SPIKES model for breaking bad news ${ }^{40}$. The sPIKES model has four goals: first, to collect information determining the patient's understanding, expectations, and readiness to receive difficult news; second, to give simplified and clear information in keeping with the patient's preferences; third, to acknowledge and support the patient's emotional reactions to the news; and fourth, to formulate a care plan in a joint effort with the patient ${ }^{40}$. Those goals can be attained by realizing the 6 steps of the sPIKEs model, which are setting up the interview, assessing the patient's perception, obtaining the patient's invitation, giving knowledge and information to the patient, addressing the patient's emotions with empathic responses, and strategizing and summarizing ${ }^{40}$. In addition to those 6 steps, we propose the inclusion of a final step called "self-care," because difficult conversations are often described as distressing and unsatisfying by physicians ${ }^{41}$. Engaging in "self-care" measures such as mindful meditation and reflective writing can be helpful in managing difficult conversations and could help to prevent compassion burnout ${ }^{42}$. Table i presents a proposed modified sPIKEss model, which can be applied in clinical settings when engaging in discussions about disease progression, which often precede the referral of patients with advanced cancer to PC services.

\section{SUMMARY}

Palliative care services extend beyond end-of-life care. Essential elements of PC include screening for and managing cancer-related symptoms, providing psychosocial care to patients and loved ones, providing education and support with respect to cancer and the patient's anticipated prognosis, and assisting in medical decision-making and advance care planning. Patients with advanced cancer should be referred to PC teams early in the course of their disease and should receive inpatient or outpatient PC services in conjunction with their usual oncologic care.

\section{Key Points}

Early referral of patients with advanced cancer to PC services is recommended. 


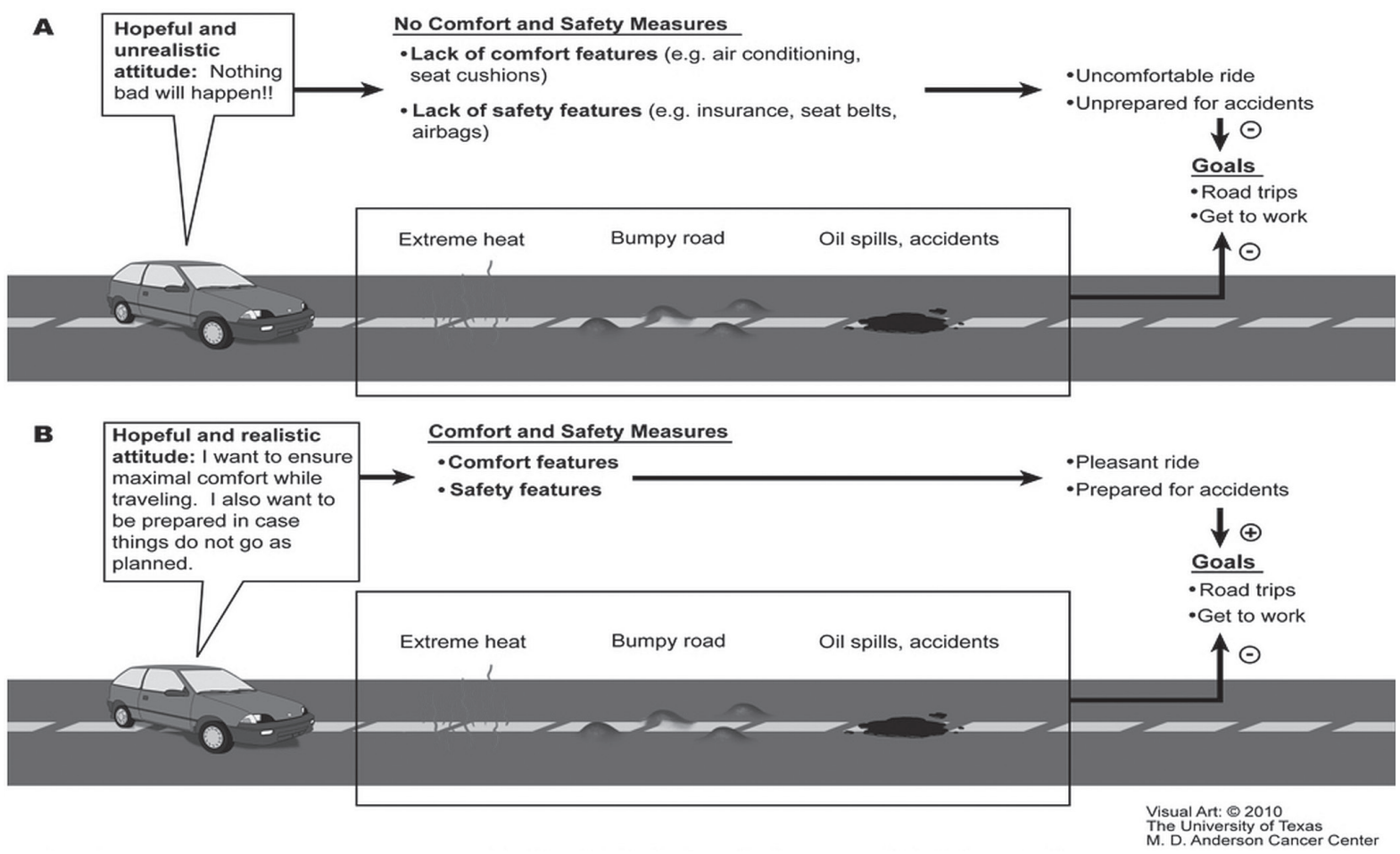

FIGURE 1 Goals for the use of a car are analogous to goals of care. (A) No comfort and safety measures. (B) Comfort and safety measures. Reprinted from Bruera and Hui, $2010^{39}$, with permission

Involvement of PC might improve symptom control and quality of life for patients.

The use of brief communication tools can facilitate discussions when referring patients to PC services.

\section{ACKNOWLEDGMENTS}

The authors give their heartfelt thanks to Tristan Williams for the formatting and final preparation of this manuscript.

\section{CONFLICT OF INTEREST DISCLOSURES}

We have read and understood Current Oncology's policy on disclosing conflicts of interest, and we declare that we have none.

\section{AUTHOR AFFILIATIONS}

*Department of Family Medicine, McGill University, Montreal, ${ }^{\dagger}$ Division of Supportive and Palliative Medicine, Royal Victoria Hospital of the McGill University Health Centre, Montreal, and ${ }^{\ddagger}$ Department of Palliative Care, Lachine Hospital Campus of the McGill University Health Centre, Lachine, QC.

\section{REFERENCES}

1. Canadian Cancer Society. Palliative Care [Web page]. Toronto, ON: Canadian Cancer Society; n.d. [Available at: http:// www.cancer.ca/en/get-involved/take-action/what-we-aredoing/palliative-care/?region=on; cited 3 August 2018]

2. Bakitas MA, Tosteson TD, Li Z, et al. Early versus delayed initiation of concurrent palliative oncology care: patient outcomes in the ENABLE III randomized controlled trial. $J$ Clin Oncol 2015;33:1438-45.

3. Zimmermann C, Swami N, Krzyzanowska M, et al. Early palliative care for patients with advanced cancer: a clusterrandomised controlled trial. Lancet 2014;383:1721-30.
4. Greer JA, Pirl WF, Jackson VA, et al. Effect of early palliative care on chemotherapy use and end-of-life care in patients with metastatic non-small-cell lung cancer. J Clin Oncol 2012;30:394-400.

5. Temel JS, Greer JA, Muzikansky A, et al. Early palliative care for patients with metastatic non-small-cell lung cancer. $N$ Engl J Med 2010;363:733-42.

6. Canadian Partnership Against Cancer (CPAC). Palliative and End-of-Life Care. Toronto, ON: CPAC; 2017. [Downloadable from: http://www.systemperformance.ca/report/palliativeend-of-life-care/; cited 8 April 2018]

7. Ferrell BR, Temel JS, Temin S, et al. Integration of palliative care into standard oncology care: American Society of Clinical Oncology clinical practice guideline update. JClin Oncol 2017;35:96-112.

8. Abernethy AP, Aziz NM, Basch E, et al. A strategy to advance the evidence base in palliative medicine: formation of a palliative care research cooperative group. J Palliat Med 2010;13:1407-1413.

9. Pirl WF, Muriel A, Hwang V, et al. Screening for psychosocial distress: a national survey of oncologists. J Support Oncol 2007;5:499-504.

10. Haun MW, Estel S, Rucker G, et al. Early palliative care for adults with advanced cancer. Cochrane Database Syst Rev 2017;6:CD011129.

11. Bagcivan G, Dionne-Odom JN, Frost J, et al. What happens during early outpatient palliative care consultations for persons with newly diagnosed advanced cancer? A qualitative analysis of provider documentation. Palliat Med 2018;32:59-68.

12. Hoerger M, Greer JA, Jackson VA, et al. Defining the elements of early palliative care that are associated with patientreported outcomes and the delivery of end-of-life care. JClin Oncol 2018;36:1096-102. 
TABLE I Modified SPIKESS model for referring patients to palliative care ${ }^{40, a}$

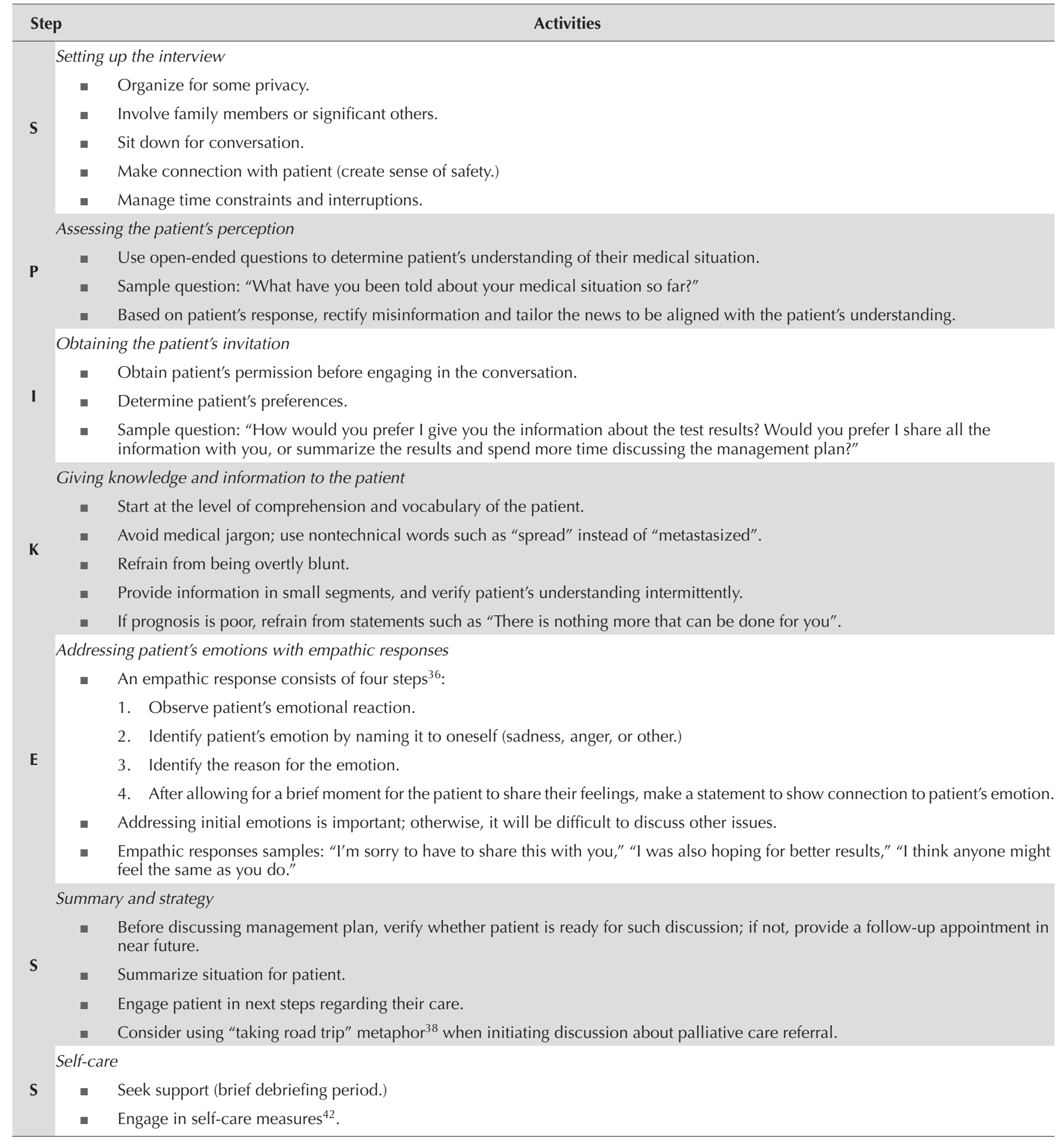

a Adapted and reprinted with permission.

13. Temel JS, Greer JA, Admane S, et al. Longitudinal perceptions of prognosis and goals of therapy in patients with metastatic non-small-cell lung cancer: results of a randomized study of early palliative care. J Clin Oncol 2011;29:2319-26.

14. World Health Organization (wHo). WHO Definition of Palliative Care [Web page]. Geneva, Switzerland: wHo; n.d. [Available at: http://www.who.int/cancer/palliative/definition/ en/; cited 6 May 2018]
15. Hui D, Kim SH, Roquemore J, Dev R, Chisholm G, Bruera E. Impact of timing and setting of palliative care referral on quality of end-of-life care in cancer patients. Cancer 2014;120:1743-9.

16. Temel JS, Greer JA, El-Jawahri A, et al. Effects of early integrated palliative care in patients with lung and GI cancer: a randomized clinical trial. J Clin Oncol 2017;35:834-41.

17. Rugno FC, Paiva BS, Paiva CE. Early integration of palliative care facilitates the discontinuation of anticancer treatment 
in women with advanced breast or gynecologic cancers. Gynecol Oncol 2014;135:249-54.

18. Yoong J, Park ER, Greer JA, et al. Early palliative care in advanced lung cancer: a qualitative study. JAMA Intern Med 2013;173:283-90.

19. United States, Department of Health and Human Services, National Institutes of Health, National Cancer Institute (NCI). NCI Dictionary of Cancer Terms: Advanced Cancer [Web resource]. Bethesda, MD: NCI; n.d. [Available at: https:// www.cancer.gov/publications/dictionaries/cancer-terms/ def/advanced-cancer; cited 4 April 2018]

20. HuiD. Prognostication of survival in patients with advanced cancer: predicting the unpredictable? Cancer Control2015;22:489-97.

21. Grudzen CR, Richardson LD, Johnson PN, et al. Emergency department-initiated palliative care in advanced cancer: a randomized clinical trial. JAMA Oncol 2016;: [Epub ahead of print].

22. Ferrell B, Sun V, Hurria A, et al. Interdisciplinary palliative care for patients with lung cancer. J Pain Symptom Manage 2015;50:758-67.

23. den Herder-van der Eerden M, van Wijngaarden J, Payne S, et $a l$. Integrated palliative care is about professional networking rather than standardisation of care: a qualitative study with healthcare professionals in 19 integrated palliative care initiatives in five European countries. Palliat Med 2018;32:1091-102.

24. Hui D, Meng YC, Bruera S, et al. Referral criteria for outpatient palliative cancer care: a systematic review. Oncologist 2016;21:895-901.

25. LeBlanc TW, El-JawahriA. When and why should patients with hematologic malignancies see a palliative care specialist? Hematology Am Soc Hematol Educ Program 2015;2015:471-8.

26. Gaertner J, Maier BO, Radbruch L. Resource allocation issues concerning early palliative care. Ann Palliat Med 2015;4:156-61.

27. Dalgaard KM, Bergenholtz H, Nielsen ME, Timm H. Early integration of palliative care in hospitals: a systematic review on methods, barriers, and outcome. Palliat Support Care 2014;12:495-513.

28. Sun V, Grant M, Koczywas M, et al. Effectiveness of an interdisciplinary palliative care intervention for family caregivers in lung cancer. Cancer 2015;121:3737-45.
29. Dionne-Odom JN, Azuero A, Lyons KD, et al. Benefits of early versus delayed palliative care to informal family caregivers of patients with advanced cancer: outcomes from the ENABLE III randomized controlled trial. J Clin Oncol 2015;33:1446-52.

30. Hannon B, Swami N, Pope A, et al. Early palliative care and its role in oncology: a qualitative study. Oncologist 2016;21:1387-95.

31. Kaasa S, Knudsen AK, Lundeby T, Loge JH. Integration between oncology and palliative care: a plan for the next decade? Tumori 2017;103:1-8.

32. Nipp RD, Greer JA, El-Jawahri A, et al. Age and gender moderate the impact of early palliative care in metastatic non-small cell lung cancer. Oncologist 2016;21:119-26.

33. Bruera E, Yennurajalingam S. Palliative care in advanced cancer patients: how and when? Oncologist 2012;17:267-73.

34. Bruera E, Hui D. Conceptual models for integrating palliative care at cancer centers. J Palliat Med 2012;15:1261-9.

35. Fallowfield L, Lipkin M, Hall A. Teaching senior oncologists communication skills: results from phase i of a comprehensive longitudinal program in the United Kingdom. J Clin Oncol 1998;16:1961-8.

36. Ptacek JT, Eberhardt TL. Breaking bad news. A review of the literature. JAMA 1996;276:496-502.

37. Mack JW, Smith TJ. Reasons why physicians do not have discussions about poor prognosis, why it matters, and what can be improved. J Clin Oncol 2012;30:2715-17.

38. Hui D, Zhukovsky DS, Bruera E. Serious illness conversations: paving the road with metaphors. Oncologist 2018;23:730-3.

39. Bruera E, Hui D. Integrating supportive and palliative care in the trajectory of cancer: establishing goals and models of care. J Clin Oncol 2010;28:4013-17.

40. Baile WF, Buckman R, Lenzi R, Glober G, Beale EA, Kudelka AP. SPIKES- a six-step protocol for delivering bad news: application to the patient with cancer. Oncologist 2000;5:302-11.

41. Fallowfield L, Jenkins V. Communicating sad, bad, and difficult news in medicine. Lancet 2004;363:312-19.

42. Kearney MK, Weininger RB, Vachon MLS, Harrison RL, Mount BM. Self-care of physicians caring for patients at the end of life: "Being connected... a key to my survival." JAMA 2009;301:1155-64. 